\title{
Étude aérothermique de l'admission d'air dans un cylindre par une méthode de description arbitraire Lagrange-Euler
}

\author{
JAMEL BessRour ${ }^{\mathrm{a}}$ \\ U.R. Mécanique Appliquée Ingénierie et Industrialisation, ENIT, BP 37, Le Belvédère, 1002 Tunis, Tunisie
}

Reçu le 23 mai 2006, accepté le 26 mars 2008

\begin{abstract}
Résumé - Une analyse numérique du processus d'évolution de l'écoulement dans le plan axial d'un cylindre d'un moteur à deux soupapes et quatre temps pendant la course d'admission est effectuée en utilisant une méthode d'éléments-finis pour un problème à frontières mobiles. Le domaine de calcul, à géométrie variable, inclut simultanément le conduit d'admission, la région de la soupape et la chambre de combustion. Le mouvement des interfaces entre le fluide, le piston et la soupape a été considéré. La méthode Lagrangienne Eulérienne Arbitraire (ALE) a été utilisée pour décrire les champs aérodynamique et thermique. Deux modèles d'interpolation des vitesses de maillage ont été adoptés : l'un est rattaché au mouvement du piston et l'autre à celui de la soupape. Le processus d'évolution et d'établissement des diverses structures tourbillonnaires et du champ thermique dans le plan axial du cylindre, caractérisé par une chambre hémisphérique et des soupapes latérales, est présenté. Cette étude a montré que la modélisation adoptée conduit à une simulation numérique avec une précision raisonnable en comparaison avec les résultats expérimentaux publiés récemment.
\end{abstract}

Mots clés : Moteur à combustion interne / admission / aérothermique / simulation numérique / méthode ALE / tumble

\begin{abstract}
Aerodynamic and thermal modelling of air intake in motored engin. We present in this paper a study on flow evolution during intake stroke in the axial plane of a two valve motored engine cylinder, using a finite-elements numerical method with movable boundary. The movable boundary computation field includes simultaneously the intake port, the inlet valve area and the combustion chamber. An arbitrary Lagrangian-Eulerian kinematics description method (ALE) is adopted to describe the flow and thermal fields, considering the interface movement between the fluid and both inlet valve and piston. The evolution and establishment process of tumbling vortical structures and thermal field in the symmetry axial plane is presented. The modelling adopted in this study leads to a reasonable precision numerical simulation in comparison with experimental results recently published.
\end{abstract}

Key words: Motored engine / intake / flow / thermal / numerical simulation / ALE method / tumble

\section{Introduction}

L'identification du champ aérodynamique et thermique à l'intérieur d'un cylindre d'un moteur à combustion interne pendant la course d'admission est une étape très importante pour la compréhension des phénomènes physiques survenant durant le cycle moteur.

Le mouvement de l'air admis dans le cylindre a une influence capitale sur les performances du moteur. Il conditionne directement le taux de remplissage, les échanges thermiques et la qualité de la combustion. Il est bien connu que la structure du champ de l'écoulement est un

\footnotetext{
a Auteur pour correspondance :

Jamel.bessrour@enit.rnu.tn
}

facteur déterminant pour l'initiation, la propagation et le rendement du processus de combustion ainsi que pour l'apparition d'émissions polluantes.

Le processus d'admission de l'air est un phénomène instationnaire complexe dans un domaine à géométrie variable (déplacement du piston et de la soupape) et son étude nécessite le couplage conduit d'admission-cylindre pour mettre en évidence la structure de l'écoulement dans la zone de la soupape caractérisée par des dimensions géométriques inférieures à l'alésage et variables dans le temps.

Les phénomènes aérodynamiques qui prennent naissance au niveau des soupapes ont été très souvent mis en évidence expérimentalement. Plusieurs essais 


\section{Nomenclature}

\begin{tabular}{|lll|}
\hline$C_{\mathrm{p}}$ & chaleur massique & $\mathrm{J} \cdot \mathrm{kg}^{-1} \cdot \mathrm{K}^{-1}$ \\
$h$ & coefficient de convection & $\mathrm{W} \cdot \mathrm{m}^{-2} \cdot \mathrm{K}^{-1}$ \\
$n$ & normale à la paroi & \\
$N$ & vitesse de rotation & $\mathrm{tr} . \mathrm{min}^{-1}$ \\
$P$ & pression & $\mathrm{Pa}$ \\
$P M H$ & point mort haut & \\
$P M B$ & point mort bas & \\
$r$ & rayon de manivelle & $\mathrm{m}$ \\
$t$ & temps & $\mathrm{S}$ \\
$T$ & température & $\mathrm{K}$ \\
$T_{v, \alpha}$ & rapport tumble & \\
$V$ & vitesse absolue & $\mathrm{m} . \mathrm{s}^{-1}$ \\
$W$ & vitesse du maillage & $\mathrm{m} \cdot \mathrm{s}^{-1}$ \\
Symboles grecs & \\
$\alpha$ & angle de rotation & $\mathrm{rd}$ \\
$\lambda$ & conductivité thermique & $\mathrm{W} \cdot \mathrm{m}^{-1} \cdot \mathrm{K}^{-1}$ \\
$\mu$ & viscosité dynamique & $\mathrm{kg} \cdot \mathrm{m}^{-1} \cdot \mathrm{s}^{-1}$ \\
$\rho$ & masse volumique & $\mathrm{kg} \cdot \mathrm{m}^{-3}$ \\
$\omega$ & vitesse angulaire & $\mathrm{rd} . \mathrm{s}^{-1}$ \\
$\Omega$ & domaine d'étude & \\
indices & & \\
$\mathrm{e}$ & air admis & \\
$\mathrm{ex}$ & extérieur & \\
$\mathrm{g}$ & gaz de combustion & \\
$i$ & nœud du maillage & \\
$\mathrm{p}$ & piston & \\
$\mathrm{pa}$ & paroi & \\
$\mathrm{s}$ & soupape d'admission & \\
$\mathrm{se}$ & soupape d'échappement & \\
$x$ & abscisse & \\
$y$ & ordonnée & \\
& & \\
\hline
\end{tabular}

expérimentaux ont été réalisés en régime d'écoulement permanent pour caractériser le mouvement de l'air induit par les soupapes [1-4] et [5,6] plus récemment. Il a été bien établi que le jet d'air à travers la soupape interagit avec les parois du cylindre pour créer de larges structures tourbillonnaires. En général, les principaux mouvements de l'air induits par le jet conique ont été caractérisés et classés en deux catégories : le mouvement de rotation induit tangentiellement dans le cylindre (swirl) et le mouvement de rotation dans le plan axial (tumble) [7]. Il est bien clair que la génération d'un écoulement avec une intense vorticité (swirl et/ou tumble) dans le cylindre pendant la course d'admission est un moyen très efficace pour obtenir une grande intensité de turbulence qui pourra se maintenir pendant la course de compression. La stabilité de ces mouvements tourbillonnaires permet ainsi de maintenir une grande turbulence pendant la phase d'injection du combustible et créer les conditions optimales pour l'initiation et le développement du processus de combustion. La forme de la chambre de combustion joue également un rôle très important quant à la structure des écoulements établis.
La caractérisation expérimentale des écoulements dans un mono-cylindre a intéressé plusieurs constructeurs moyennant certaines techniques de visualisation et l'utilisation de cylindres transparents [8-12]. L'insuffisance des moyens de mesure a souvent rendu difficile l'étude détaillée de l'évolution du processus des écoulements.

Récemment, le développement des techniques basées sur la vélocimétrie par images de particules (PIV) a motivé les constructeurs pour étudier l'évolution dans le temps de la structure de l'écoulement dans le cylindre [13-18]. Une étude récente [19] a permis d'identifier la nature des écoulements instantanés pendant la phase d'admission et de compression d'un mono-cylindre à deux soupapes par une technique de PIV bi-dimensionnelle.

La deuxième approche, basée sur la modélisation numérique et la simulation de ces écoulements complexes, a intéressé plusieurs auteurs depuis deux décennies. La plupart ont essayé de contourner les difficultés numériques, moyennant des hypothèses simplificatrices autour du modèle géométrique et en utilisant le plus souvent la méthode des volumes-finis. La simulation numérique permet de prédire les résultats en faisant varier largement les paramètres du problème et les conditions aux limites, une démarche bien plus difficile sur le plan expérimental. Par contre, l'approche numérique a toujours posé des difficultés liées à la nature instationnaire des écoulements et à la complexité du domaine à géométrie variable qui induisent des temps de calcul très longs et exigent des calculateurs à très hautes capacités mémoire. Plusieurs travaux sur les écoulements dans les cylindres de moteurs à combustion interne ont été élaborés et présentés. Certains auteurs ont introduit des simplifications considérables au processus dont notamment l'assimilation de la soupape à un simple disque fixe ou en mouvement [20-25] ou la considération d'un cylindre axisymétrique à soupape axiale [26-28]. Certains de ces travaux n'ont pas eu de support expérimental alors que d'autres ont présenté des validations expérimentales très grossières. Plus récemment, le développement important des outils de calcul a permis d'améliorer la précision et la qualité des simulations numériques [29-31] basées souvent sur la méthode des volumes-finis notamment par le biais de codes de calcul de type CFD [32].

Ainsi, notre travail porte essentiellement sur la simulation numérique des champs aérodynamiques et thermiques dans le plan axial de symétrie d'un cylindre à deux soupapes. Notre intérêt a porté essentiellement sur l'identification des mouvements rotatifs d'axe horizontal, en rouleau (tumble), particulièrement générés par la majorité des moteurs à essence. Notre objectif est de développer et tester une méthodologie numérique basée sur la méthode des éléments-finis par le biais d'un code de calcul polyvalent et ouvert (CASTEM $2000 \mathrm{du}$ CEA) [33] de type boîte à outils offrant de nombreuses fonctionnalités et dont la structure évolutive permet de développer des procédures personnalisées. L'avantage de la méthode des éléments-finis est de permettre les formulations avec des couplages fluide-structure tels que notamment le traitement du problème aérothermique couplé 
avec la résolution de l'équation de la chaleur dans la structure du piston-cylindre.

Pour tenir compte de la variabilité du domaine et donc de la déformation du maillage, nous avons voulu tester la méthode « Lagrangienne Eulérienne Arbitraire» (ALE). Pour la discrétisation spatiale du domaine fluide, on veut que les éléments-finis des frontières du domaine coïncident constamment avec les particules de fluide (formulation lagrangienne) alors qu'au sein du domaine et afin de pouvoir gérer les grands déplacements du fluide, on adopte un taux de déformation du maillage différent et indépendant de celui du fluide. Le maillage se déforme à la vitesse des particules de fluide sur les frontières mobiles du domaine (piston et soupape). Le reste du domaine se déforme avec une vitesse obtenue par un modèle d'interpolation entre la vitesse de la paroi mobile et la vitesse nulle des parois fixes de façon à conserver une bonne qualité du maillage. Pour cela, nous avons considéré deux sous-domaines : le premier, rattaché au mouvement du piston, concerne la surface balayée par celui-ci avec une vitesse de maillage obtenue par une interpolation linéaire. Le deuxième domaine comporte le conduit d'admission et la zone de la soupape dont la vitesse instantanée est déterminée à partir de la loi de la came de commande. Nous avons adopté, pour ce domaine, une vitesse de maillage interpolée par un laplacien avec des conditions de frontières appropriées. Concernant le problème thermique, nous avons adopté des conditions initiales et aux limites qui essayent de reproduire d'une façon réaliste les phénomènes physiques.

La validation d'une méthode numérique avec un domaine fluide en mouvement est toujours difficile à cause de l'inexistence de solutions analytiques. Les champs aérodynamiques obtenus à diverses positions du piston sont comparés aux résultats des essais obtenus sur le moteur expérimental qui a servi de support à l'analyse numérique, publiés par Huang [19]. Les solutions semblent bonnes d'un point de vue physique, avec une précision qui peut être considérée comme relativement correcte étant donné le grand nombre de paramètres numériques et expérimentaux qui interfèrent dans cette étude.

\section{Description du cas d'étude}

Le moteur utilisé comme support pour la présente étude est un mono-cylindre à quatre temps et deux soupapes ayant un diamètre de $52,4 \mathrm{~mm}$, une course de $57,8 \mathrm{~mm}$ et un volume engendré de $124,6 \mathrm{~cm}^{3}$. La chambre de combustion est hémisphérique et les soupapes, placées de part et d'autre de l'axe central, commandées par un arbre à came en tête, présentent un plan de symétrie axial. La soupape d'admission ayant une course de 7,25 $\mathrm{mm}$ et une inclinaison de $20^{\circ}$, a une avance à l'ouverture de $30^{\circ}$ et un retard à la fermeture de $80^{\circ}$.

Il s'agit des caractéristiques du moteur expérimental (Fig. 1) conçu avec les modifications nécessaires pour l'installation d'un dispositif expérimental [19] support d'une vélocimétrie par images de particules (PIV). Les essais ont été effectués à une vitesse $N=1500$ tr.min $^{-1}$.

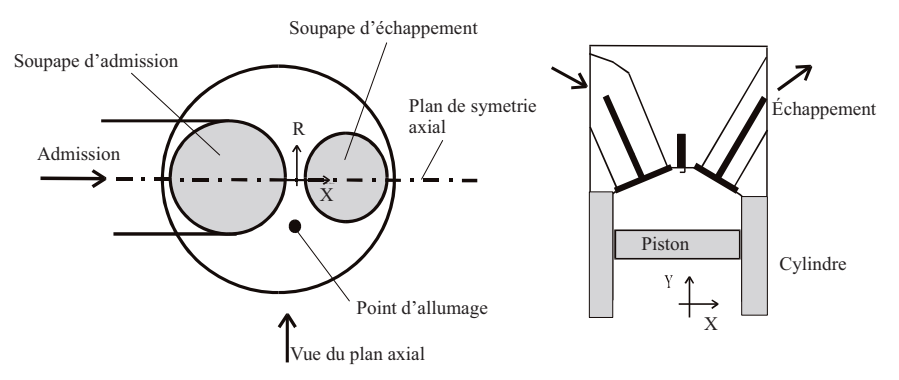

Fig. 1. Schéma du moteur.

\section{Méthodologie numérique}

\subsection{Position du problème}

Les équations moyennes qui gouvernent un écoulement instationnaire et incompressible d'un fluide newtonien dérivent des principes de conservation de la masse, de la quantité de mouvement (Navier-Stokes) et de l'énergie. L'étude envisagée concernant la course d'admission d'air avec des mouvements du piston et de la soupape pose un problème de variabilité de la géométrie dans le temps. Pour cela, il est nécessaire d'adopter une technique de régénération du maillage à chaque pas du temps. Une description eulérienne, lagrangienne, arbitraire (ALE) est bien appropriée pour décrire ce problème d'écoulement multidimensionnel à frontières mobiles et qui peut être éventuellement couplé avec un problème structure.

La formulation ALE permet de combiner les avantages de la représentation lagrangienne (bonne définition des frontières variables, facilité d'imposer des conditions aux limites matérielles) et ceux de la formulation eulérienne (possibilité de prise en compte de grandes distorsions). Le principe de cette formulation mixte est le développement de maillage se déplaçant à taux indépendant de celui de la matière. En conséquence, la matière se déplace par rapport au maillage, comme dans une formulation eulérienne pure, mais la forme des frontières est contrôlée par des conditions aux limites du problème, comme dans une formulation lagrangienne. Le fait que le système de référence de calcul ne soit ni fixe dans l'espace (formulation eulérienne), ni calculé comme résultat d'une déformation directement liée à celle de la matière (formulation lagrangienne), procure une grande liberté dans la formulation du problème qui fera intervenir la vitesse du maillage notée $\vec{W}$.

Afin de limiter, dans une première approche, les grandes difficultés numériques que cette analyse va rencontrer, on a considéré les hypothèses suivantes :

- Le fluide est l'air, incompressible, visqueux et newtonien, et l'écoulement est bi-dimensionnel et laminaire;

- Les propriétés du fluide sont constantes et l'effet de la gravité est négligé;

- Une condition de non-glissement est imposée dans les interfaces fluide-parois mobiles;

- L'énergie de dissipation est négligée dans l'équation de l'énergie. 
Dans ces conditions, les équations qui gouvernent la formulation ALE font apparaître dans le terme de convection la vitesse absolue de déplacement des nouds du maillage $\vec{W}$ :

- Équation de continuité :

$$
\operatorname{div} \vec{V}=0
$$

- Équation des quantités de mouvement :

$$
\rho\left(\frac{\partial \vec{V}}{\partial t}+(\vec{V}-\vec{W}) \overrightarrow{\operatorname{grad}}(\vec{V})\right)=-\overrightarrow{\operatorname{grad}} P+\mu \Delta \vec{V}
$$

- Équation de l'énergie :

$$
\frac{\partial T}{\partial t}+(\vec{V}-\vec{W}) \overrightarrow{\operatorname{grad}}(T)=\frac{\lambda}{\rho C_{\mathrm{p}}} \Delta T
$$

L'équation de l'énergie est traitée en couplage séquentiel faible avec les équations de Navier-Stokes.

\subsection{Configuration et conditions aux limites}

La modélisation géométrique utilisée comme support à l'analyse numérique est constituée de la chambre de combustion (délimitée par la face supérieure du piston, des parois du cylindre et de la soupape d'échappement en position de fermeture) et du conduit d'admission (délimité par la soupape d'admission et les parois fixes) considérés avec les dimensions réelles du moteur. En effet, le couplage conduit d'admission-cylindre est nécessaire pour appréhender d'une façon réaliste la nature des écoulements dans la zone du siège de soupape de dimension très inférieure à celle de l'alésage et variable dans le temps.

Pour tenir compte de la variabilité de la géométrie due aux mouvements simultanés du piston et de la soupape, nous avons subdivisé le domaine d'étude $\Omega_{t}$ en deux sousdomaines $\Omega_{1}$ et $\Omega_{2}$ (Fig. 2) de topologies différentes et discrétisés séparément en éléments-finis.

Le domaine $\Omega_{1}$ est rattaché au piston et se déforme suivant le mouvement de celui-ci avec un maillage structuré, constitué uniquement de quadrangles à 4 nœuds. Le domaine $\Omega_{2}$, constitué du conduit d'admission et de la zone de la soupape d'admission va se déformer en fonction du mouvement de celle-ci. Le maillage de $\Omega_{2}$ est constitué de quadrangles et de triangles. L'interface commune à ces deux domaines $\Delta_{1-2}=\left(\Omega_{1} \cap \Omega_{2}\right)$ sera naturellement fixe dans le temps (vitesse de maillage nulle).

Moyennant cette configuration, les conditions aux limites considérées et les vitesses du maillage sont les suivantes.

\subsubsection{Zone balayée par le piston $\Omega_{1}$}

\section{Cinématique :}

- Sur AB : $V_{x}(t)=0 ; V_{y}(t)=V_{\mathrm{p}}(t)$ (vitesse instantanée du piston) ;

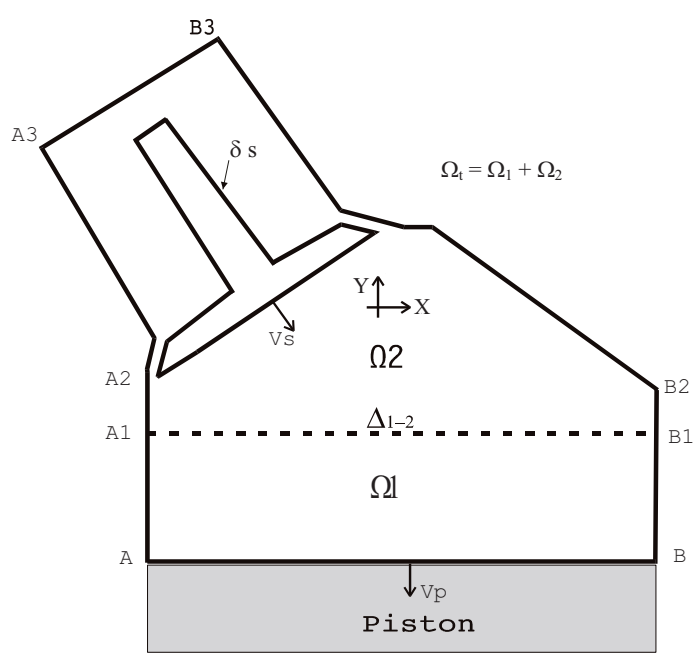

Fig. 2. Schéma du domaine d'étude.

- Sur $\mathrm{AA}_{1}$ et $\mathrm{BB}_{1}: V_{x}(t)=0 ; V_{y}(t)=0$;

- Le piston, entraîné par un système bielle-manivelle tournant à la vitesse angulaire $\omega$ a un mouvement périodique. En négligeant les harmoniques d'ordres supérieurs, la vitesse instantanée du piston en fonction de l'angle de rotation $\alpha=\omega t$ est sinusoïdale : $V_{\mathrm{p}}(t)=\omega r \sin \omega t$

- Conditions initiales à $t=0$ : piston au PMH et pression $P=0$.

\section{Thermique :}

- Sur AB : température imposée : $T(t)=T_{\mathrm{p}}$ (température du piston);

- Sur $\mathrm{AA}_{1}$ et $\mathrm{BB}_{1}: \frac{\mathrm{d} T}{\mathrm{~d} n}=h\left(T-T_{\mathrm{pa}}\right)$ : le cylindre est refroidi et le flux est échangé avec l'extérieur, à travers les parois, par convection;

- Conditions initiales à $t=0: T(0)=T_{\mathrm{g}}$ (température des gaz de combustion qui subsistent dans le cylindre après la fermeture de la soupape d'échappement).

\section{Vitesse du maillage $\boldsymbol{W}$ :}

- Sur $\mathrm{AB}: W(t)=V_{\mathrm{p}}(t)$ (description lagrangienne : vitesses des nouds confondues avec les vitesses des particules);

- Sur $\Delta_{1-2}: W(t)=0$;

- Sur $\mathrm{AA}_{1}, \mathrm{BB}_{1}$ et le reste de $\Omega_{1}$ : on considère une variation linéaire de $W$ depuis la valeur nulle sur $\Delta_{1-2}$ jusqu'à $V_{\mathrm{p}}$ sur $\mathrm{AB}$.

\subsubsection{Zone de la soupape $\Omega_{2}$}

\section{Cinématique :}

- Sur $\mathrm{A}_{1} \mathrm{~A}_{2} \mathrm{~A}_{3}$ et $\mathrm{B}_{1} \mathrm{~B}_{2} \mathrm{~B}_{3}: V_{x}(t)=0$ et $V_{y}(t)=0$;

- Sur $\delta S$ (contour de la soupape) : $V(t)=V_{\mathrm{s}}(t)$ : vitesse instantanée de la soupape déterminée à partir de la loi de levée de la soupape dont l'ouverture se fait avec une avance de $30^{\circ}$ et la fermeture avec un retard de $80^{\circ}$ (angle total d'ouverture : $290^{\circ}$ ) représentée par la figure 3 ; 


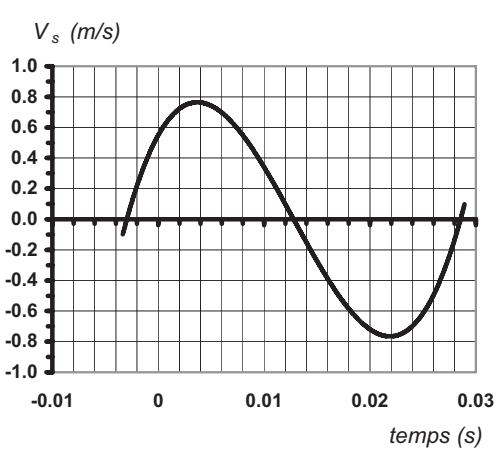

Fig. 3. Vitesse instantanée de la soupape.

- Conditions initiales à $t=0$ : le piston au PMH et la soupape est partiellement ouverte à $30^{\circ}$.

\section{Thermique :}

- Parois du cylindre $\mathrm{A}_{1} \mathrm{~A}_{2}$ et $\mathrm{B}_{1} \mathrm{~B}_{2}$ : refroidissement par convection;

- Soupape d'échappement : température imposée : $T(t)=T_{\mathrm{se}}$

- Contour de la soupape $\partial S: \frac{\mathrm{d} T}{\mathrm{~d} n}=0$;

- Conduit d'admission : $T(t)=T_{\mathrm{e}}$ (température de l'air admis) ;

- Conditions initiales à $\mathrm{t}=0: T(0)=T_{\mathrm{g}}$ dans la chambre de combustion et $T(0)=T_{\mathrm{e}}$ dans le conduit.

\section{Vitesse du maillage $\mathrm{W}$ :}

- Sur le contour extérieur $\partial \Omega_{2}: W(t)=0$;

- Sur le contour intérieur $\delta S: W(t)=V_{\mathrm{s}}(t)$;

- À l'intérieur du domaine $\Omega_{2}$ : nous avons adopté une méthode simple, couramment utilisée, d'interpolation de la vitesse de maillage par la résolution d'une équation au laplacien $\Delta W=0$ avec les conditions de frontière appropriées (entre la valeur nulle et $V_{\mathrm{s}}$ ).

La méthode de génération du maillage utilisée, sans faire appel à des critères plus évolués d'optimisation de certains paramètres de qualité de maillage, présente l'avantage de la simplicité et peut se révéler suffisante pour l'étude envisagée.

\subsection{Méthode numérique}

Pour résoudre, en instationnaire, les équations nonlinéaires de Navier-Stokes couplées avec l'équation de l'énergie, nous avons utilisé la méthode des élémentsfinis qui a l'avantage de pouvoir inclure facilement des maillages de géométries complexes et de permettre des calculs couplés avec des domaines structures. Nous avons intégré la méthodologie numérique présentée dans le code de calcul polyvalent et ouvert, CASTEM $2000 \mathrm{du}$ CEA, offrant plusieurs fonctionnalités. Ce code a l'avantage de permettre le développement, moyennant un langage interne, de procédures personnalisées spécifiques au problème à résoudre.
La discrétisation spatiale, dans le domaine $\Omega_{t}$, des équations de conservation en formulation mixte (vitesse, pression) et des conditions aux limites est obtenue à partir d'une formulation variationnelle de Galerkin en utilisant un opérateur de discrétisation des équations de Navier-Stokes dans une description ALE, moyennant la définition à chaque pas de temps d'un champ de vitesse $W$ des nouds du maillage. Pour notre cas, ce champ est indépendant des vitesses des particules et ne dépend que des vitesses instantanées de la soupape et du piston. Le maillage est d'abord construit de macro-éléments triangles et quadrangles (interpolation linéaire pour les vitesses, pression constante par élément) qui sont découpés en quatre éléments linéaires. L'assemblage des contributions de tous les éléments conduit finalement à un système matriciel où les inconnues sont les vecteurs globaux contenant les valeurs nodales des vitesses et de la pression faisant intervenir la matrice de la divergence et sa transposée dite matrice du gradient ainsi que les matrices de masse, de convection et de diffusion. Ces matrices, calculées sur le domaine spatial discrétisé $\Omega_{t}$, doivent être recalculées à chaque pas du temps. Cette complexité par rapport au cas eulérien, nécessite d'apporter un grand soin à la discrétisation temporelle du problème et justifie les difficultés rencontrées pour assurer la consistance et la convergence en temps de l'algorithme.

La discrétisation en temps est abordée par une méthode de différences finies. La discrétisation spatiale au temps $t^{n}$ donne lieu à un vecteur global $x^{n}$ (contenant les coordonnées des nœuds de vitesse et de pression) discrétisé aux pas de temps entiers $n$ alors que les inconnues $V$ et $P$ sont discrétisées aux demi-pas de temps $n-\frac{1}{2}$. Cette façon de procéder permet une intégration du déplacement du maillage, précise à l'ordre 2 en temps, de manière simple [33]. Les quantités calculées aux temps $n-\frac{1}{2}$ sont évaluées sur le maillage estimé $x^{n-\frac{1}{2}}$ (moyenne entre $x^{n}$ et $\left.x^{n-1}\right)$. Ainsi, au pas de temps $n$ donné, on dispose des quantités $x^{n}, x^{n-1}, V^{n-\frac{1}{2}}$ et $W^{n-\frac{1}{2}}$. On passe au pas de temps suivant $n+1$ en commençant par résoudre l'équation qui permet de déplacer les nœuds de vitesse et de pression du maillage en calculant $x^{n+\frac{1}{2}}$ à partir de $x^{n}$, $W^{n+\frac{1}{2}}$ et du pas de temps $\Delta t^{n}$. Dans cette étape, seuls les nœuds du maillage sont modifiés alors que les types et connectivités des éléments restent inchangés, ce qui constitue un des avantages de la méthode ALE par rapport à une méthode où on remaillerait ce qui impliquerait une projection des inconnues discrétisées d'un maillage à un autre, source d'erreurs numériques. On utilise par la suite une discrétisation temporelle des équations de Navier-Stokes par une méthode semi-implicite (implicite sur la pression, explicite sur les autres variables), l'avancée en temps se faisant par un schéma de type Euler et moyennant un schéma de convection décentré. Pour résoudre le système discrétisé, on commence par calculer la pression en prenant la divergence de l'équation matricielle de conservation des quantités de mouvement (ce qui permet d'éliminer l'inconnue $V^{n+\frac{1}{2}}$ dont la divergence est nulle) et en résolvant le système obtenu par une méthode directe de type Choleski. Une fois le système résolu, on 


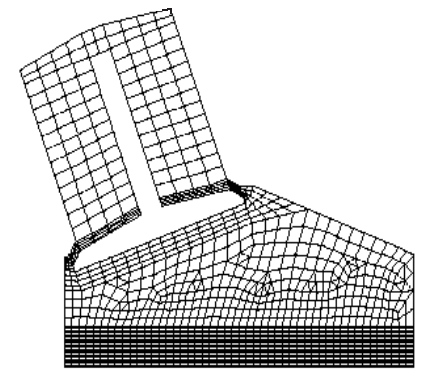

$\alpha=0^{\circ}$

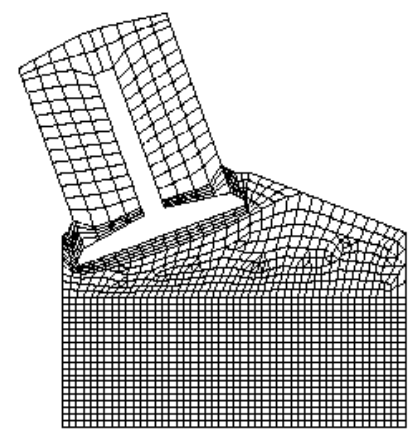

$\alpha=90^{\circ}$

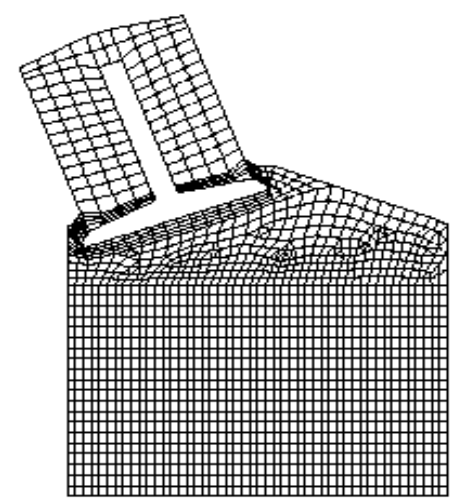

$\alpha=180^{\circ}$

Fig. 4. Maillage.

remplace la pression par sa valeur dans l'équation des quantités de mouvement et on calcule $V^{n+\frac{1}{2}}$.

Il faut noter que la discrétisation explicite des termes de convection et de diffusion impose une limitation sur le pas de temps afin d'assurer des conditions de stabilité. Il faut ajouter également une limitation du pas de temps afin d'assurer la non-dégénérescence du maillage. En effet, le passage d'un maillage donné à l'instant suivant doit se faire en déplaçant uniquement les nœuds avec les contraintes suivantes :

- le nombre et le type d'éléments ne doivent pas varier;

- les connectivités doivent rester identiques;

- les éléments ne doivent pas dégénérer (triangles changeant d'orientation, quadrangles devenant concaves...).

Pour ces raisons complexes, le pas de temps a été appréhendé d'une façon directe par des estimations qui ont conduit à en fixer une valeur suffisamment petite pour assurer la convergence.

L'équation de l'énergie est résolue séquentiellement en deuxième étape, moyennant un schéma de convection décentré et un schéma explicite en temps. Le pas de temps adopté après quelques essais est $10^{-6} \mathrm{~S}$

La démarche de résolution peut se résumer ainsi :

- on définit le maillage optimal initial à $t=0$;

- on initialise les variables à leurs valeurs initiales;

- pour un pas de temps $\Delta t$ :

- on calcule les vitesses instantanées du piston $V_{\mathrm{p}}$ et de la soupape $V_{\mathrm{s}}$ utilisées dans les conditions aux limites du problème dynamique;

- on détermine les vitesses du maillage $W$ dans $\Omega_{1}$ (interpolation linéaire) et dans $\Omega_{2}$ interpolation par un laplacien);

- on réactualise les coordonnées des nouds en utilisant l'opérateur « Forme » pour le déplacement du maillage et on contrôle la distorsion de celui-ci ;

- on résout les équations (1) et (2);

- on substitue les vitesses dans l'équation (3) pour obtenir le champ de température $T$;
- on continue au pas de temps suivant à $(t+\Delta t)$ en mettant à jour les nouvelles conditions aux limites $\left(V_{\mathrm{p}}\right.$ et $V_{\mathrm{s}}$ ) et les vitesses du maillage $W$.

\section{Résultats numériques}

\section{1 Évolution aérothermique dans le plan de symétrie central}

On démarre les simulations de la course d'admission à $t=0$ lorsque le piston est au PMH et la levée de la soupape est de $1 \mathrm{~mm}$ (ouverture à $30^{\circ}$ avant le $\mathrm{PMH}$ ). La vitesse de rotation utilisée pour les essais est de 1500 tr.min ${ }^{-1}$, soit une durée de course de $10^{-2}$ s et un nombre de pas de temps de $10^{4}\left(\Delta t=10^{-6} \mathrm{~s}\right)$. La vitesse maximale du piston est de $4,5 \mathrm{~m} . \mathrm{s}^{-1}$ et sa vitesse moyenne est $2,9 \mathrm{~m} \cdot \mathrm{s}^{-1}$. La levée de la soupape atteint sa valeur maximale $(7,25 \mathrm{~mm})$ avec une vitesse nulle pour $\alpha=115^{\circ}$. Elle est d'environ $5 \mathrm{~mm}$ en fin de course, le piston étant au PMB. La figure 4 représente le maillage en position initiale $\left(\alpha=0^{\circ}\right)$, médiane $\left(\alpha=90^{\circ}\right)$ et finale $\left(\alpha=180^{\circ}\right)$. Le maillage a été affiné autour des soupapes. Le nombre de nœuds retenus est d'environ 1700 afin de limiter la taille du problème qui devient rapidement très exigeant en mémoire et en temps de calcul.

Les résultats présentés constituent l'aboutissement de nombreux essais numériques. Malgré le fait que nous nous sommes intéressés principalement à l'écoulement dans le plan axial pour appréhender la création du mouvement tourbillonnaire « Tumble », la résolution a nécessité environ 12 heures de calcul sur un PC de 2,4 GHz. Pour faire apparaître les détails de l'écoulement, le maillage doit être serré notamment au niveau de la soupape et l'utilisation d'un pas de temps inadéquat conduit facilement à une divergence des calculs.

Les résultats (Fig. 5) permettent d'analyser le mouvement de l'air pendant la phase d'admission, d'identifier la structure de l'écoulement intimement liée à la levée de la soupape et d'apprécier les effets dus à la géométrie particulière caractérisée par une chambre hémisphérique et une soupape et conduit inclinés. 


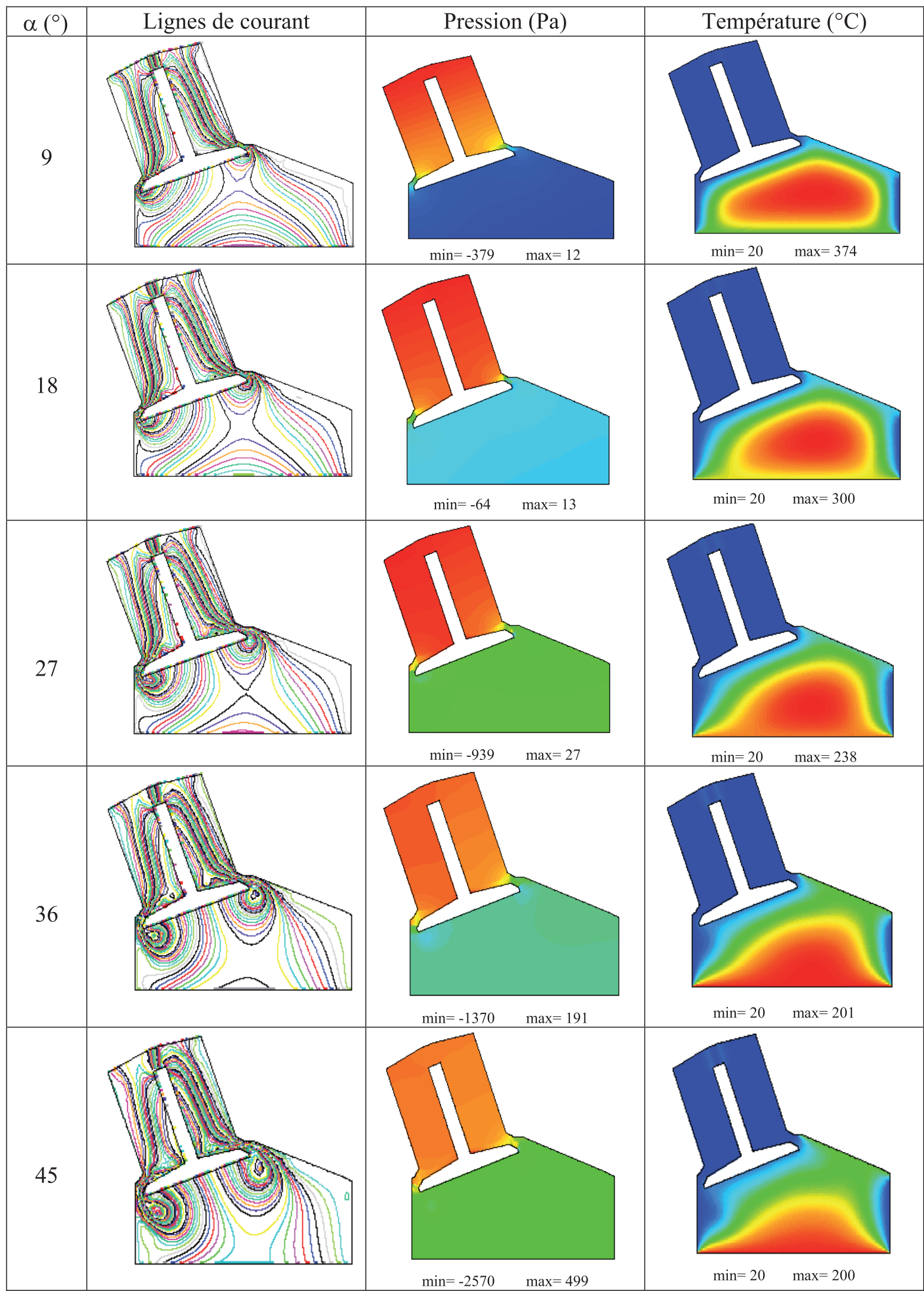

Fig. 5. Évolution des lignes de courant, pressions et températures dans le plan de symétrie axial pendant la course d'admission (pas de variation de $\alpha: 9^{\circ}$ ). 


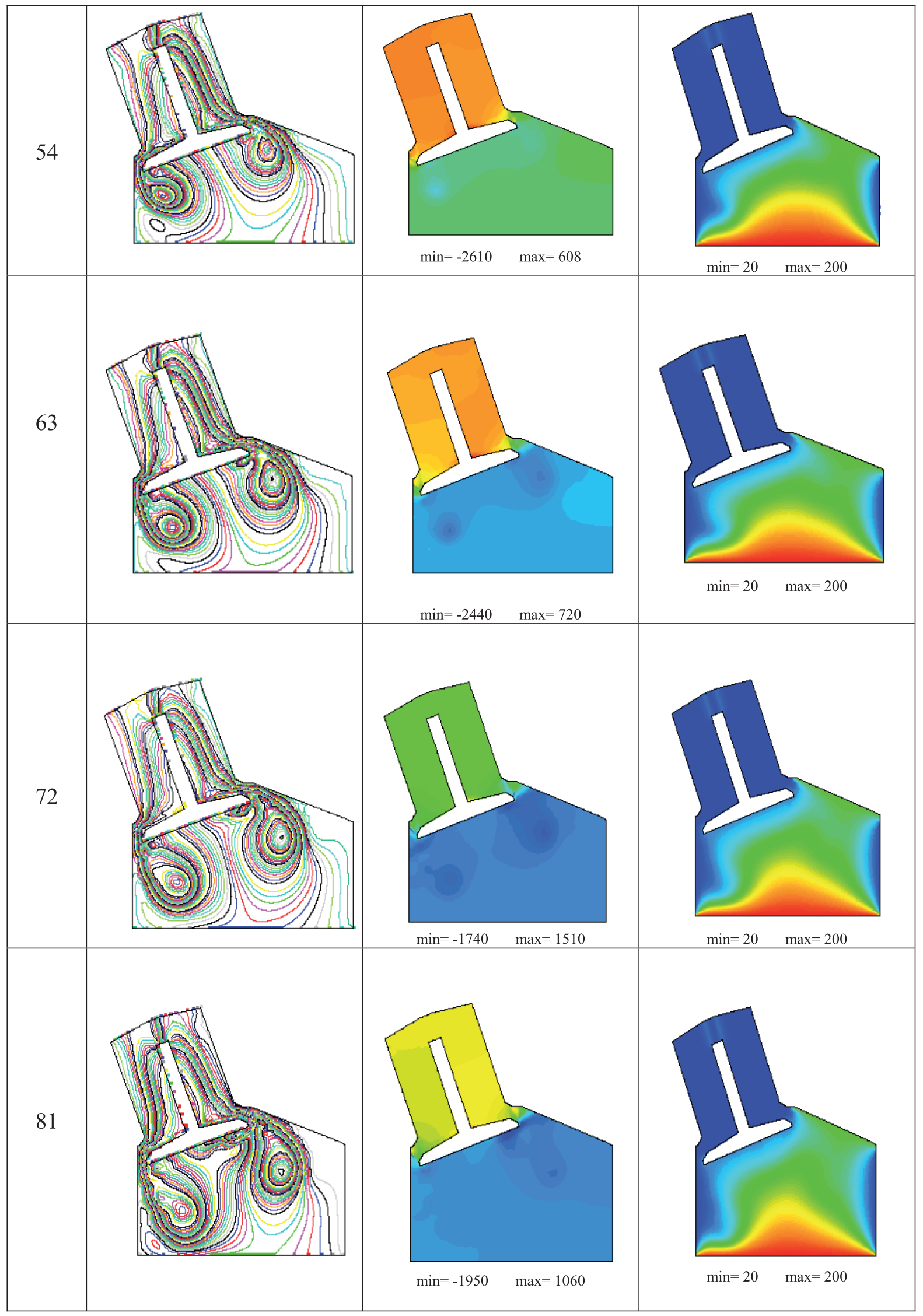

Fig. 5. Suite. 


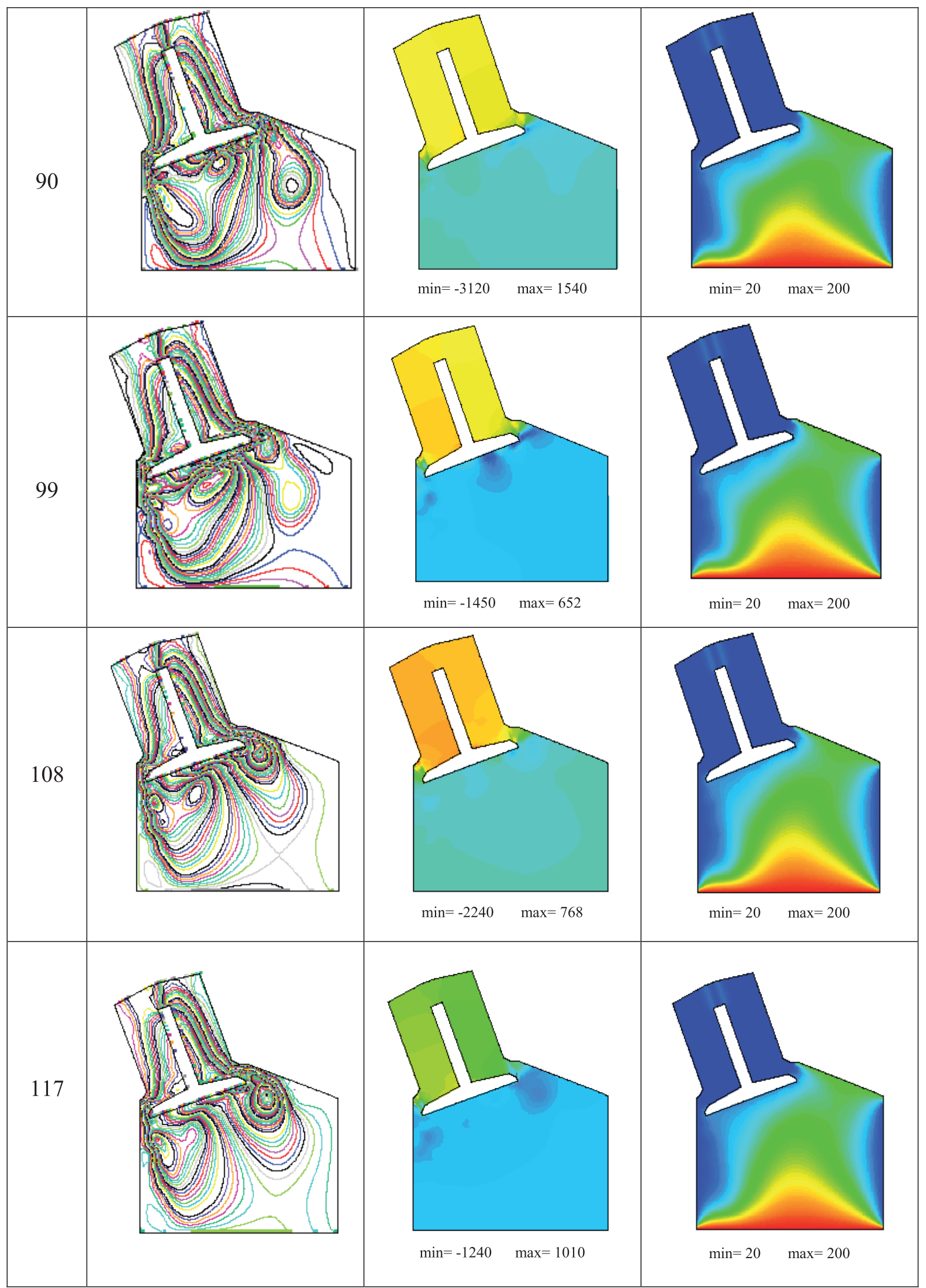

Fig. 5. Suite. 


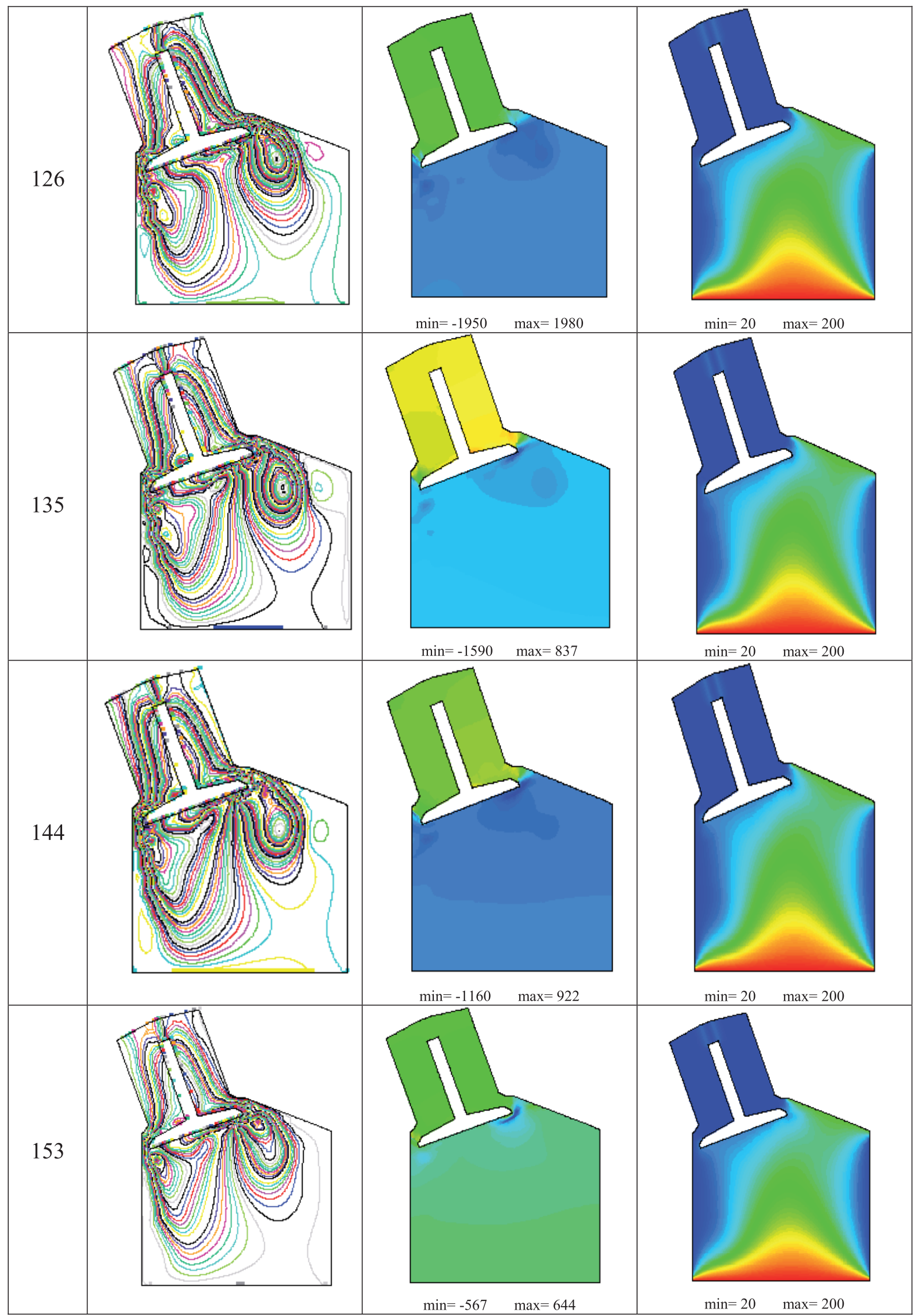

Fig. 5. Suite. 


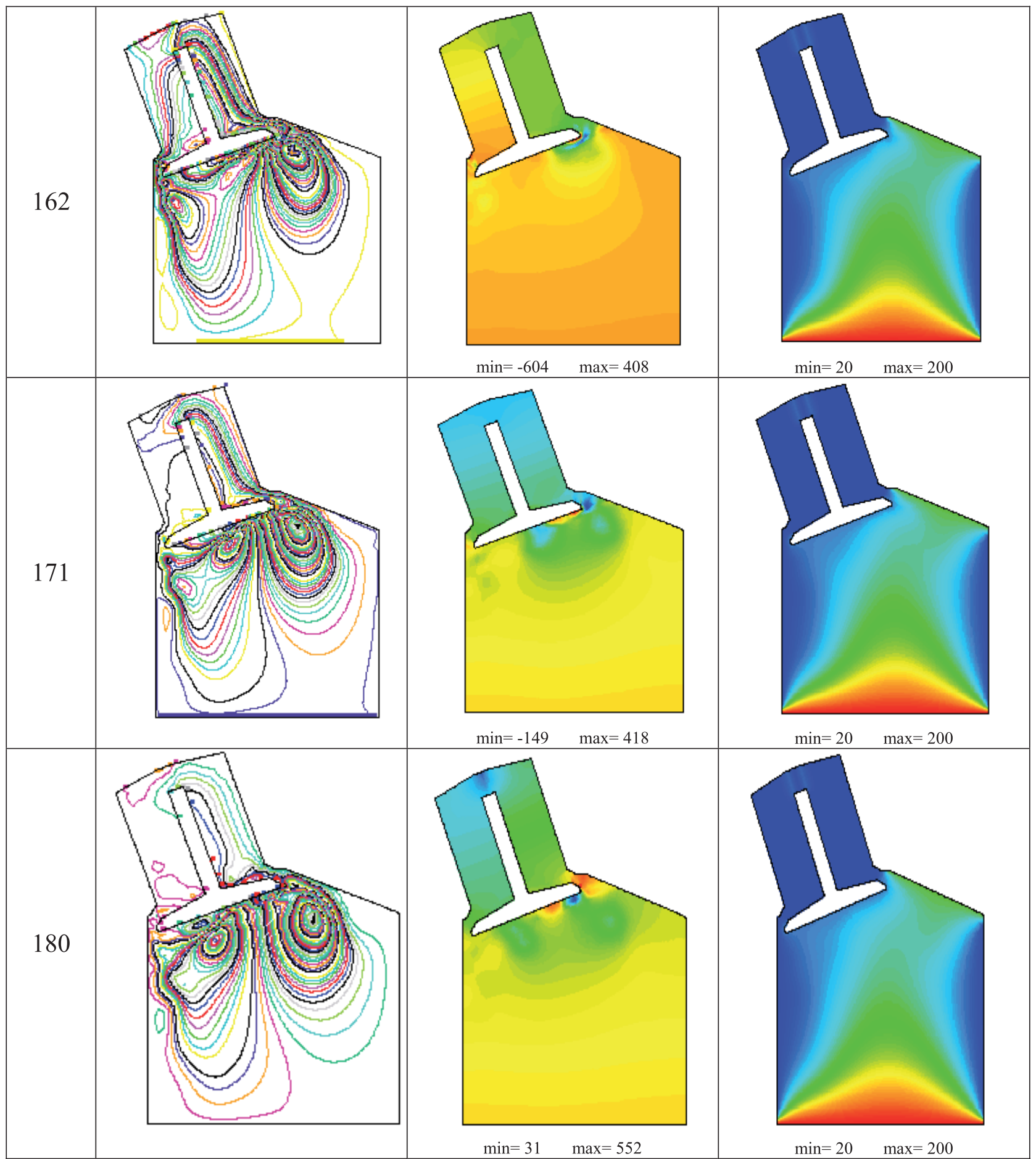

Fig. 5. Suite.

On note bien que l'ouverture de la soupape induit un jet conique avec des gradients de vitesse localement importants qui atteignent leur maximum lorsque la vitesse instantanée du piston est maximale $\left(\alpha=90^{\circ}\right)$ et fait apparaître également des dépressions locales accentuées.

L'interaction du jet d'air avec les parois du cylindre, le piston et la soupape conduit à la formation de deux tourbillons non symétriques de sens opposés qui conservent globalement une stabilité jusqu'à la fin de course. Au départ, une partie de l'écoulement est dirigée le long de la paroi droite du cylindre et pointe vers la tête du piston. Une partie de cet écoulement subit un retour vers la soupape. Ce phénomène est en accord avec les résultats théoriques et expérimentaux publiés 
concernant l'écoulement à travers un obstacle de type disque : une zone de recirculation se forme dans la zone proche du sillage de l'obstacle. Il est bien évident que la zone dépressionnaire générée en bas de la soupape entraîne des courants de retour vers la soupape et la formation d'une structure de recirculation rotative dans la zone supérieure droite du cylindre.

Jusqu'à $\alpha=90^{\circ}$, la vitesse du piston augmente et l'ouverture de la soupape est encore partielle. L'écoulement à travers la partie gauche de la soupape n'est pas très fort au départ et les particules pointent vers la tête du piston avant de former également une zone tourbillonnaire. L'influence du jet gauche va devenir progressivement importante : l'écoulement le long de la paroi gauche du cylindre tourne en bas et rejoint l'écoulement montant du vortex primaire pour former un vortex de sens contraire. Les deux tourbillons formés vont grossir avec l'augmentation de la vitesse du piston et le vortex gauche va évoluer progressivement pour se scinder en deux vortex décentrés adjacents qui vont durer jusqu'à la fin de la course.

À partir de l'angle $\alpha=90^{\circ}$, la vitesse du piston commence à diminuer et les lignes de courant qui pointent vers le piston vont diminuer d'intensité pour renforcer les tourbillons formés. Pendant cette phase, des zones mortes vont apparaître avec la formation localement d'un petit tourbillon instable notamment à droite de la soupape d'échappement et en bas de la paroi gauche du cylindre.

Ainsi, la structure d'un vortex de type «tumbling » est formée dans le plan de symétrie central du cylindre. Un contrôle peut être effectué à chaque pas de temps pour vérifier la conservation du volume (volume admis égal au volume déplacé par le piston). La vitesse maximale de l'air au passage de la soupape atteint $90 \mathrm{~m} . \mathrm{s}^{-1}$. Pendant cette phase, le volume balayé par le piston sera constamment en dépression par rapport au conduit d'admission. Cette dépression est localement importante au centre du vortex et sa valeur maximale atteint environ $-3000 \mathrm{~Pa}$. La connaissance de cette pression et de la température locale permettrait d'évaluer la densité de l'air admis et d'estimer le taux de remplissage du cylindre.

La figure 5 représente également l'évolution du champ de température dans le cylindre. Jusqu'à environ $20^{\circ}$, la température maximale, proche de $300{ }^{\circ} \mathrm{C}$, est localisée au niveau des gaz chauds résiduels au centre de la chambre de combustion. Le mélange progressif avec l'air frais introduit à travers la soupape conduit à une diminution de la température au centre du cylindre. La température est plus faible au contact avec les parois du cylindre sous l'effet de l'échange convectif avec les parois. Les vortex qui ont pris naissance contribuent également à une diminution locale de la température du mélange. Pendant la majeure partie de la course, la température maximale correspond à la zone en contact avec le piston (environ $200^{\circ} \mathrm{C}$ ) et avec la soupape d'échappement, mal refroidie. En fin de course, la température moyenne de la masse d'air admis est d'environ $60^{\circ} \mathrm{C}$. Ainsi, l'échauffement de l'air frais admis est dû partiellement au mélange avec les gaz résiduels et principalement à l'effet des parois chaudes.

\subsection{Analyse quantitative du mouvement d'écoulement dans le cylindre}

Afin de quantifier les mouvements d'écoulements identifiés dans le plan de symétrie, un rapport tumble $T_{\mathrm{v}, \alpha}$ a été défini, pour chaque angle $\alpha[18]$ :

$$
T_{\mathrm{v}}, \alpha=\frac{\sum_{i=1}^{n}\left(\frac{\partial v_{y}}{\partial x}-\frac{\partial v_{x}}{\partial y}\right)_{i}}{2 n \omega}
$$

Le numérateur représente la somme des vorticités déterminées pour un ensemble de $n$ nœuds du domaine d'indices $i, \omega$ étant la vitesse angulaire. Ce coefficient peut être interprété comme le rapport de la vitesse angulaire moyenne des vortex par la vitesse angulaire moyenne de l'arbre et caractérise ainsi l'intensité du mouvement tourbillonnaire.

La détermination de ce rapport se fait en posttraitement à partir du rotationnel des vecteurs vitesses instantanées calculées pour chaque angle $\alpha$. Elle a été limitée au cylindre (domaine $\Omega_{1}$ ). Les résultats montrent que le module de ce coefficient est faible en début de course et augmente par la suite avec l'angle $\alpha$ pour atteindre ses valeurs maximales à partir de $90^{\circ}$. En effet, c'est à partir de cet angle, correspondant à la vitesse maximale du piston, que les structures des vortex se développent. Le module maximal atteint est de $-0,15$. Cette valeur est relativement faible et trouve son interprétation par le fait qu'aucun dispositif particulier n'a été introduit dans cette application pour accentuer la formation des structures tumble. Des dispositifs tels que les soupapes à déflecteurs ou l'utilisation d'une plaque plane horizontale dans le plan de symétrie du conduit d'admission munie d'un papillon, adoptés sur des moteurs similaires, conduisent à des rapports tumble plus importants, proches de $-0,5$.

\section{Comparaisons aux données expérimentales}

Pour contrôler la qualité de la simulation numérique et la prédiction de l'allure générale de l'écoulement, nous avons comparé les résultats du champ aérodynamique moyen obtenus à diverses positions du piston avec les résultats des essais obtenus par la PIV sur le moteur expérimental retenu comme support à l'analyse numérique, publiés par Huang [19]. En fait, l'objectif étant uniquement la visualisation des écoulements, le moteur à cylindre transparent est entraîné par un moteur électrique à 1500 tr.min ${ }^{-1}$ et aspire uniquement de l'air. Les auteurs de cette étude ont utilisé une étude de PIV bidimensionnelle pour visualiser et numériser d'une façon entièrement découplée les écoulements dans un plan diamétral et dans un plan axial de symétrie. Il faut noter que le champ de visualisation de l'écoulement dans le plan axial qui nous intéresse ne couvre pas la totalité de la surface balayée par le piston et que les zones proches des soupapes et des parois latérales restent à l'extérieur du champ de visualisation de la caméra $\left[4,6 \times 4,6 \mathrm{~cm}^{2}\right]$. 


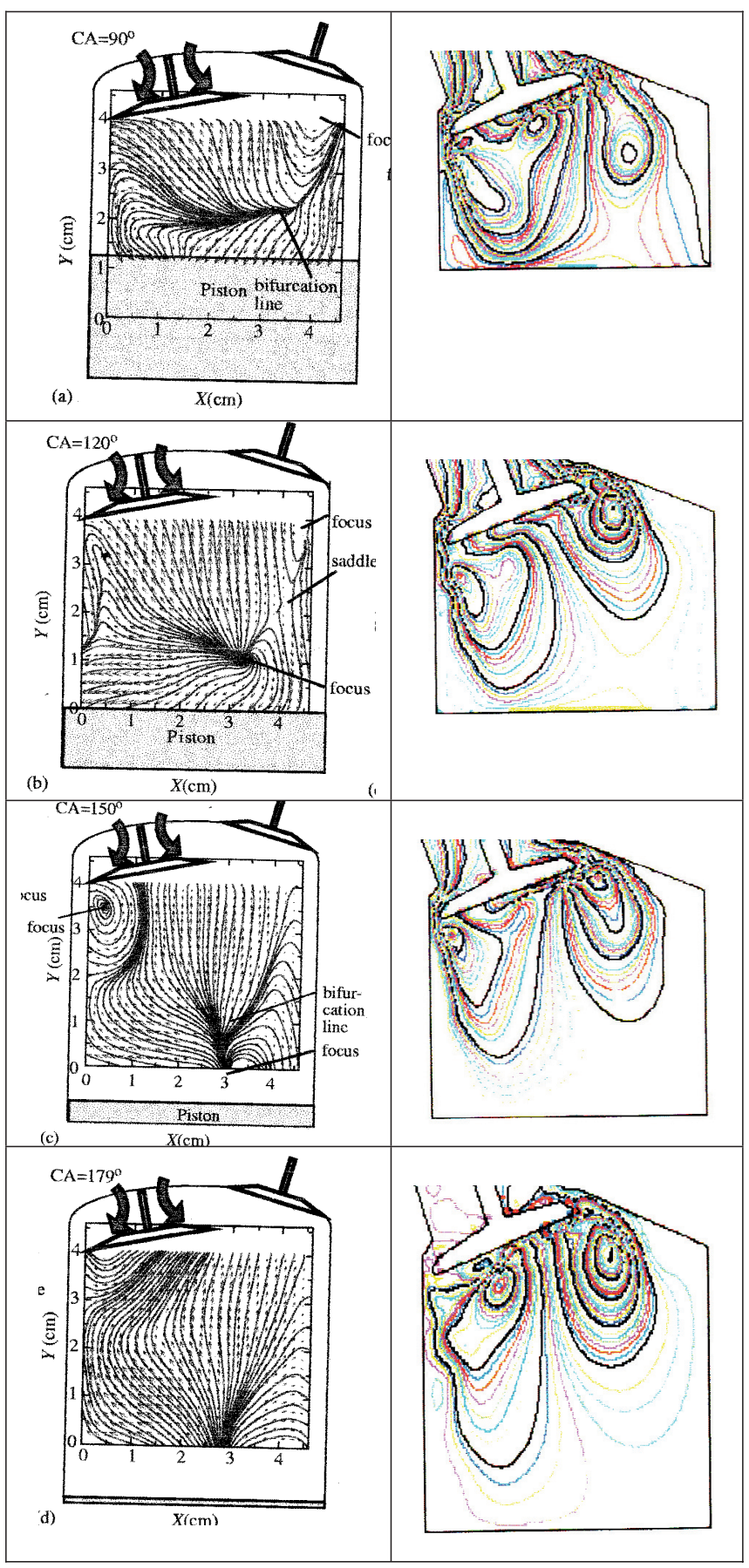

Fig. 6. Lignes de courant expérimentales [19] (gauche) et numériques (droite).

La figure 6 représente une comparaison des résultats numériques et expérimentaux dans le plan axial, en termes de lignes de courant, pour quatre positions du piston. Pour $\alpha=90^{\circ}$, on constate que la similitude est assez bonne concernant l'apparition du tourbillon à droite du cylindre et l'apparition de la zone de bifurcation avec les lignes de courant qui pointent vers le piston et celles qui remontent vers la soupape, contournent le tourbillon et redescendent vers le piston.

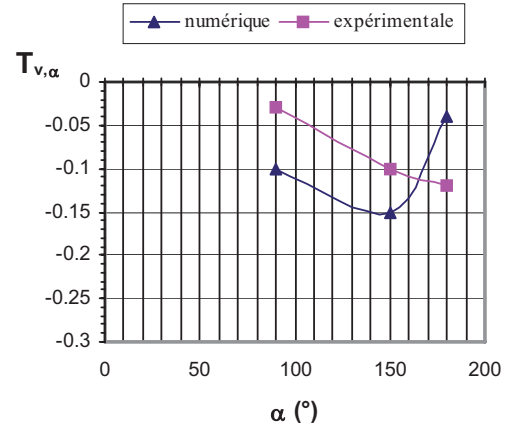

Fig. 7. Variation du rapport Tumble avec l'angle de rotation.

La position $\alpha=120^{\circ}$ est caractérisée par l'enregistrement d'un vortex instable localisé dans la région droite en bas qui entraîne la formation d'un « saddle » à quatre voies qui sépare deux principales lignes d'écoulement de sens opposés. Les résultats numériques concordent bien au niveau de l'écoulement autour du vortex latéral et du vortex gauche mais ne font pas apparaître clairement le vortex instable signalé. L'absence de lignes de courant dans la zone correspondante prouve qu'il est nécessaire d'affiner localement le maillage et l'analyse pour pouvoir détecter son éventuelle apparition.

Pour $\alpha=150^{\circ}$, le vortex instable se déplace vers le bas en suivant le mouvement du piston et se trouve à l'extérieur du champ de vision de la caméra. Le « point saddle » disparaît, mais les principales lignes de courant vers le haut restent bien visibles. Le centre du vortex gauche est bien mis en évidence. Les résultats numériques témoignent d'une grande similitude notamment au niveau de la zone de bifurcation et de l'établissement des structures tourbillonnaires. On note également que le vortex instable près du piston n'apparaît pas et que la zone correspondante est vide de lignes de courant. Les causes peuvent être identiques à celles citées précédemment. Cette structure de l'écoulement va durer jusqu'à la fin de la course d'admission $\left(\alpha=180^{\circ}\right)$ avec les mêmes similitudes et constats.

Il faut noter que pour chaque angle $\alpha$, les lignes de courant expérimentales sont obtenues par une technique d'interpolation à partir des vecteurs vitesses obtenus sur une moyenne d'une centaine d'images. Les lignes de courant numériques sont obtenues sur des nœuds limités en nombre et différents de ceux adoptés pour la visualisation et suite à un traitement numérique basé sur plusieurs hypothèses simplifiant le modèle évoquées précédemment. Ces facteurs peuvent constituer les causes majeures des écarts relatifs observés dans la comparaison qui a surtout mis en relief une concordance dans le processus d'établissement des grands vortex.

Pour les mêmes causes, la comparaison des rapports $T_{\mathrm{v}, \alpha}$ (Fig. 7) pour certains angles ne peut convenir qu'à dégager les tendances d'évolution de ce paramètre. En plus, les écarts observés sont amplifiés par le fait que les valeurs expérimentales sont, à chaque position, déterminées par une moyenne sur l'ensemble des points d'application des vitesses identifiées à l'intérieur 
du champ de visualisation de la caméra qui ne couvre qu'un carré de 4,6 cm de côté alors que les dimensions du domaine de calcul sont plus grandes (course : $5,78 \mathrm{~cm}$ et diamètre : $5,24 \mathrm{~cm})$.

\section{Conclusions}

En général, cette étude a montré que les simulations numériques, par le biais du modèle adopté, conduisent à une identification de la structure de l'écoulement dans le plan axial d'un cylindre pendant la course d'admission avec une précision physiquement raisonnable en comparaison avec les résultats expérimentaux. Elle a permis, notamment, de mettre en évidence le processus d'établissement des diverses structures tourbillonnaires dans un cylindre à chambre de combustion hémisphérique et soupapes latérales.

Bien que la plupart des études numériques similaires publiées utilisent la méthode des volumes-finis, cette étude montre qu'il est possible de développer une méthodologie numérique, à travers un code polyvalent, basée sur la méthode des éléments-finis en utilisant une description ALE, avec un temps de calcul qui reste raisonnable si la taille du problème est relativement limitée. L'avantage de la méthode des éléments-finis est de pouvoir appréhender plus facilement des géométries complexes et de permettre les couplages avec les problèmes de structures.

Il faut bien noter que la qualité des résultats dépend naturellement de la finesse du maillage mais surtout qu'il faut apporter une attention bien particulière au pas de temps pour éviter les problèmes d'instabilité et de divergence.

L'avantage de la méthode ALE est la structuration de l'algorithme en procédures bien séparées (résolution des équations de Navier-Stokes découplée du déplacement du maillage). Par contre, toutes les matrices du système dépendent du temps et doivent être recalculées à chaque pas ce qui augmente le temps de calcul, et la qualité des résultats (distorsion du maillage, convergence, stabilité) est très sensible au pas de temps adopté.

La résolution de l'équation de l'énergie avec la même méthodologie numérique et les conditions aux limites adoptées permet d'appréhender raisonnablement le champ de température de l'air en fin d'admission. La connaissance de ce champ de température et la détermination du champ de pression permettent d'évaluer la densité de l'air en fin d'admission et le taux de remplissage du cylindre.

Enfin, l'implémentation de la description ALE dans un code de calcul par éléments-finis et son approfondissement avec notamment la recherche d'algorithmes plus avancés pour le déplacement du maillage et l'amélioration des temps de calcul, peut offrir un outil intéressant pour la recherche et le développement dans le domaine de l'aérodynamique interne des moteurs.

\section{Références}

[1] C. Arcoumanis, A.F. Bicen, J.H. Whitelaw, Measurement in a motored four stroke reciprocating model engine, J. Fluids Eng. 104 (1982) 104-235

[2] K.Y. Kang, R.D. Reitz, Intake flow structure and swirl generation in a four-valve Diesel engine, Spring Technical Conference, ASME 1999, 32-2. Paper No. 99-ICE-182, (1999)

[3] P. Snauwaert, R. Sierens, Experimental study of the swirl motion in direct injection Diesel engines under steady state flow conditions, SAE 860026, 1986

[4] G. Tippelmann, A new method of investigation of swirl ports, SAE 770404,1977

[5] J. Gazeaux, D.G. Thomas, Caractérisation du mouvement de rotation de l'air en écoulement stationnaire dans un mono-cylindre Diesel en fonction des conditions d'admission, Entropie 234 (2001) 13-19

[6] J.-E.Yun, New evaluation indices for bulk motion of incylinder flow trough intake port system in cylinder head, Proc. ImechE, Part D: J. Automobile Eng. 216 (2002) 513-S21

[7] J.B. Heywood, Fluid motion within the cylinder of internal combustion engines, ASME J. Fluids Eng. 109 (1987) 3-35

[8] A. Ekchian, D.P. Hoult, Flow visualization study of the intake process of an internal combustion engine, SAE paper 790095, Warrendale, PA, USA, 1979

[9] T. Hirotimi, I. Nagayama, S. Kobayashi, T. Yamamasu, Study of induction swirl in a spark ignition engine, SAE paper 810496, Warrendale, PA, USA, 1981

[10] C. Arcoumanis, A.F. Bicen, N.S. Vlachos, J.H. Whitelaw, Effects of flow and geometry boundary conditions on fluid motion in a motored IC model engine, Proc. Institution Mech. Eng., 196, 1982, pp. 1-10

[11] T. Liou, D.A. Saltavicca, Cycle resolved LDV measurements in a motored IC engine, ASME J. Fluids Eng. 107 (1985) 232-240

[12] S. Nadarajah, S. Balabani, M.J. Tindal, M. Yianneskis, The effect of swirl on the annular flow past an axisymmetric poppet valve, Proc. Institution Mech. Eng., Part C, 212, 1998, pp. 473-484

[13] M. Raffer, C.E. Willert, J. Kompenhans, Particle Image Velocimetry - A Practical Guide, Springer, Berlin, 1998

[14] R. Adrian, Particle-imaging techniques for experimental fluid mechanics, Annual Rev. Fluid Mech. 23 (1991) 261304

[15] B. Khalighi, Study of the intake tumble motion by flow visualization and particle tracking velocimetry, Exp. Fluids 10 (1991) 230-236

[16] J. Lee, P.V. Farrel, Intake valve flow measurements of an IC engine using particle image velocimetry, SAE paper 930480, Warrendale, PA, USA, 1993

[17] G. Valentino, D. Kaufman, P.V. Farrel, Intake valve flow measurement using PIV, SAE paper 932700, Warrendale, PA, USA, 1993

[18] E. Rouland, A. Floch, A. Ahmed, D. Dionnet, M. Trinite, Characterization of intake generated tumble flow in 4valve engine using cross-correlation particle image velocimetry, Proc. Eighth International Symposium on Flow Visualization, 1998, pp. 195.1-195.15

[19] R.F. Huang, C.W. Huang, H.S. Yang, T.W. Lin, W.Y. Hsu, Topological flow evolutions in cylinder of motored 
engine during intake and compression strokes, J. Fluids Struct. 20 (2005) 105-127

[20] A.D. Gosman, Y.Y. Tsui, A.P. Watkins, Calculation of three dimensional air motion in model engines, SAE 840229, 1984

[21] T. Wakisaka, Y. Shimamoto, Y. Issihiki, Threedimensional numerical analysis of in- cylinder flows in reciprogating engines, SAE 860464, 1986

[22] W. Brandstätter, R.J.R. Johns, G. Wigley, The effect of inlet port geometry on in- cylinder flow structure, SAE 850499, 1985

[23] H. Schapertons, F. Thiele, Three dimensional computations for flowfields in DI piston bowls, SAE 860464, 1986

[24] T. Wakisaka, Y. Shimamoto,Y. Issihiki, Threedimensional numerical analysis of in-cylinder flows in reciprogating engines, SAE 860464, 1986

[25] S. Aita, A. Tabbal, G. Munk, N. Monmayeur, Y. Takenka, Y. Aoyagi et al., Numerical simulation of swirling portvalve-cylinder flow in Diesel engine, SAE 910263, 1991

[26] S. Raghay, A. Hakim, Simulation numérique d'un écoulement dans un moteur alternatif, Entropie 206 (1997) 33-42
[27] A. Giovanni, F. Karaginnis, Caractérisation des grosses structures rotationnelles de l'écoulement dans le cylindre du moteur alternatif par la méthode des tourbillons aléatoires, Entropie 139 (1988) 48-58

[28] Y. Mao, M. Buffat, D. Jeandel, Simulation of the turbulent flow inside the combustion chamber of a reciprocating engine with a finite element method, J. Fluid Eng. 116 (1994) 363-369

[29] A. Chen, A. Veshagh, S. Wallace, Intake flow predictions of a transparent DI Diesel engine, SAE 981020, 1998

[30] B. Dillies, A. Ducamin, L. Lebrere, F. Neveu, Direct injection Diesel engine simulation: a combined numerical and experimental approach fro aerodynamics to combustion, SAE 970880, 1997

[31] I. Celik, I. Yavuz, A. Smirnov, Large eddy simulations of in-cylinder turbulence for internal combustion engines: a review, Int. J. Engine Res. 2 (2001) 119-148

[32] F. Payri, J. Benajes, X. Margot, A. Gil, CFD modeling of the in-cylinder flow in direct-injection Diesel engines, Comp. Fluids 33 (2004) 995-1021

[33] S. Gounand, Rapport DMT/97/, CEA/Saclay 